\title{
Zur assyrischen Vokalharmonie und zum Wortakzent des Akkadischen ${ }^{1}$
}

\author{
Carsten Peust
}

\section{Summary}

It is argued that the so-called Assyrian vowel harmony affects stressed short $a$ in open syllables rather than unstressed $a$ as has traditionally been believed. Departing from this assumption, the accentuation rules of the Assyrian dialect of Akkadian are reconsidered. It turns out that the accent of Assyrian Akkadian largely resembles the "Hebrew type" with stress most often falling on the last stem syllable of nouns and verbs.

Im assyrischen Dialekt des Akkadischen wird in offener Silbe stehendes kurzes $a$ häufig an ein $e, i$ oder $u$ der Folgesilbe assimiliert. Da allerdings nicht jedes solche $a$ betroffen ist, nimmt man einschränkend an, dass die Vokalharmonie nur auf unbetontes $a$ wirke: „Assyr[isch] wird kurzes unbetontes $a$ dem darauf folgenden Endungsvokal angeglichen“ (GAG $\$ 10 \mathrm{e}) ;$ „...) dass in unbetonter geschlossener Silbe stehendes $a>u$ wird, sobald die Silbe durch Anfügung eines $u$-enthaltenden Afformativs geöffnet wird“ (Lewy 1921: 26); „L'attrazione vocalica [...] è solo regressiva e si verifica soltanto per la vocale $a$, quando questa è breve, non accentata e in sillaba aperta“ (Lancellotti 1962: \$112f). Von anderen wird die Bedingung noch weiter eingeschränkt, nämlich auf nichterste Silben: „Unter dem Einfluss eines nachfolgenden, kurzen oder langen, $e$, $i, u$ wird kurzes, unbetontes, in offener Silbe (welches nie die erste des Wortes ist) stehendes $a$ durch (regressive) Vokalassimilation zu $e, i, u^{\text {“ }}$ (Deller 1959: 145), oder auf Nachtonsilben: „Kurzes, in geöffneter Nachtonsilbe stehendes $a$ wird an den ihm folgenden Vokal angeglichen“ (GKT \$10a); „The post-tonic short $a$ of an open syllable is assimilated to the following short vowel“ (SNAG $\$ 2.4 .5) ; ~, a$ in a short medial syllable which follows a stressed syllable assumes the quality of the following vowel" (Buccellati 1997: 25).

Ein kritischer Punkt in der herkömmlichen Erklärung der Vokalharmonie mittels des Wortakzents ist der, dass unsere Kenntnis der akkadischen Betonung keineswegs gut abgesichert ist. In der älteren, besonders deutschen Assyriologie hat sich ein Modell der akkadischen Betonung entwickelt, das sich, wie Knudsen (1980) in einem kritischen Artikel dargestellt hat, weniger

1 Mein Dank für hilfreiche Hinweise gilt Andreas Fuchs (Tübingen), Svetlana Gasimova (Tübingen) und last but not least Daniel Schwemer (London), der den Artikel reviewt und eine Reihe konstruktiver Vorschläge gemacht hat. 
an tatsächlicher akkadischer Evidenz als an den damaligen Vorstellungen über den Wortakzent des klassischen Arabisch orientiert hat, über den jedoch seinerseits keine Sicherheit herrscht. In den aktuellen akkadischen Grammatiken wird dieses Standardmodell heute ohne Begründung, aber auch ohne Zweifel vorgetragen (z.B. GAG \$38; Huehnergard 1997: 3f.). Es stimmt übrigens auch mehr oder minder mit der Default-Akzentzuweisung überein, die man im Deutschen für unbekannte Fremdwörter anzuwenden pflegt, und mag daher vielen Assyriologen intuitiv naheliegend erscheinen. In dieses Bild passt, dass Reiner (1966: 38f.) stattdessen für das Akkadische einen durchgängigen Initialakzent erwogen hat (wie im Ungarischen; Erica Reiner war ungarische Muttersprachlerin).

Als meines Wissens einziger hat Postgate (1974: 274) die oben beschriebene Auffassung zur assyrischen Vokalharmonie in Frage gestellt: „we may wonder whether in fact an accented short $a$ in an open syllable might be liable to vowel assimilation, and not [...] the 'unbetonte' $a^{\text {" }}$ (zu seiner Begründung siehe unten Abschnitt 12). Dieser nach meiner Auffassung zutreffende Ansatz wurde weder von ihm selbst noch von nachfolgenden Grammatikern weiter verfolgt.

Ich möchte das Thema der assyrischen Vokalharmonie im Folgenden noch einmal neu aufrollen mit dem Ziel, auf dieser Basis neue, sprachinterne Anhaltspunkte für die Bestimmung des akkadischen Wortakzents zu gewinnen. Im Einzelnen zeigt die Vokalharmonie mancherlei Schwankungen, weshalb ich meine Argumentation möglichst auf gut bezeugte Wort- und Flexionsformen beschränke und sporadische Einzelgraphien ignoriere.

In zweisilbigen Wörtern mit kurzem $a$ in der ersten Silbe unterbleibt die Vokalharmonie in den meisten Fällen, so in rabi "groß (stat. constr.) “ (GKT \$63c); qadi „mit" (CAD Q 47f.); balu „ohne“ (CAD B 70f.); mati „wann?“ (CAD M I 407f.); akē „wie? (neuassyr.)“ (CAD K 322); ūmu mašil „Mittag“ (Cancik-Kirschbaum 1996: 223); alik „geh!“ (UGM \$82.1; Parpola 1984: 4f.); amur „sieh!“ (GKT \$89a); grundsätzlich auch in Stativen wie naši „trägt“ (Parpola 1974: 7; UGM \$81.4); laqi „hat genommen“ (UGM \$80.3); nadi „liegt“ (GKT \$97c); masi ,ist genug“ (CAD M I 345-7; GKT \$95e); tadin „ist gegeben“" (GAG \$102m; GKT \$100b; SNAG \$3.14.1); malik ,ist König“ (in dem häufigen Personennamen Aššur-malik, z.B. Hecker et al. 1998: 383, Michel \& Garelli 1997: 301); habul, ,ist schuldig“ (GKT \$72a). Die Vokalharmonie greift jedoch, wenn es sich bei der letzten Silbe um eine Kasusendung handelt: $k u l-u$ „jeder (Nom.)“, Akk. kal- $a$, Gen. kil-i (GKT \$62b). Auch wenn die letzte Silbe ein Possessivsuffix ist, scheint Vokalharmonie einzutreten. Das Substantiv für "Mund, Ausspruch“, dessen Stamm wohl als pa anzusetzen ist, finden wir nämlich in altassyrischen Eigennamen in den Formen $p u-s ̌ u-k e ̄ n$,sein-Ausspruchist-fest" und $i k \hat{u}(n)$-pa-ša "fest-ist-ihr-Ausspruch“ (Belege z.B. in Hecker et al. 1998: 379ff.; Michel \& Garelli 1997, Index; Stephens 1944, Index; siehe auch GAG \$65i); vgl. weiter noch pi-i „meinen Mund (Akk.)“ (Hirsch 1972: 410) 
und pi-ki „deinen(f) Mund“ (CAD P 457). Gegenüber den Formen aus Personennamen, denen ich im Zweifel eine höhere Altertümlichkeit unterstellen würde, sind in altassyrischen Texten daneben auch andere Formen belegt, die sich nicht hierzu fügen und verschiedene Ausgleichserscheinungen darzustellen scheinen. So findet man (GKT \$\$10c, 62c) für "seinen Mund (Akk.)“ alle drei Vokalisationen $p u$-šu (mit Vokalharmonie), $p a-s ̌ u$ (ohne Vokalharmonie) und pi-šu (die im Babylonischen dominierende Form).

Bezüglich der Vokalharmonie fallen die Zweisilbler also in zwei Klassen, von denen die Klasse $k u l-u$ / $p u$-šu mit dem bisherigen Standardmodell nicht erklärbar ist und als Ausnahme behandelt werden muss. Wenn wir den Unterschied zwischen beiden Klassen mit dem Wortakzent in Zusammenhang bringen wollen, so wären a priori zwei Szenarien denkbar: (1) Der Akzent fällt normalerweise auf die vorletzte Silbe, doch Kasusendungen und Suffixe sind betont, so dass die Vokalharmonie das unbetonte (vortonige) $a$ betrifft (rábi, qádi; aber kal-ú > kul-ú, pa-šú >pu-šú). (2) Der Ton fällt normalerweise auf die letzte Silbe, ausgenommen Kasusendungen und Suffixe, so dass die Vokalharmonie das betonte $a$ betrifft ( rabí, qadí; aber kál-u>kúl-u, pá-šu > pú-šu).

Nehmen wir zunächst die Gültigkeit von Szenario (1) an. Wenn Kasusendungen also grundsätzlich betont wären, wäre in Dreisilblern etwa eine Aussprache rabi'-ú „groß $\aleph^{\prime \prime}$, labir-ú „alt" und šani'-ú „der zweite, andere“ anzusetzen. Da die genannten Wörter aber keine Vokalharmonie zeigen (Belege im CAD), wäre weiter zu postulieren, dass $a$ nur in der unmittelbar vortonigen Silbe von der Vokalharmonie betroffen wäre und nicht weiter vorne im Wort, was schon recht kompliziert ist. Wenn weiter Possessivsuffixe grundsätzlich betont wären, wäre die Form „alles von ihm“ als * kal-u-šú anzusetzen und Vokalharmonie demnach hier nicht zu erwarten; es heißt jedoch $k u l-u$-šu (GKT $\$ 62 \mathrm{~b})$.

Ich schließe daraus, dass Szenario (1) nicht zu einem plausiblen Theoriegebäude führt und somit Szenario (2) das wahrscheinlichere ist. Wir wollen also dem Vorschlag Postgates folgen, wonach die assyrische Vokalharmonie betontes kurzes $a$ in offener Silbe betrifft. Damit ist auch die Beobachtung gut vereinbar, dass maximal ein $a$ pro Wort der Vokalharmonie unterliegt, denn es kann nur eine Silbe pro Wort akzentuiert sein. (Dies ist eine gängige Definition des Wortakzents: Wortakzent ist eine Eigenschaft, die eine Silbe pro Wort auszeichnet.) Eine typologische Parallele bildet der $i$-Umlaut vieler germanischer Sprachen, der ebenfalls gerade den Tonvokal betrifft (z.B. althochdeutsch gabéran „gebären“, 3.sg. gabírit „gebiert" mit Umlaut é >í).

Auf dieser Basis lassen sich jetzt für das Assyrische die folgenden Akzentregeln gewinnen:

(1) Wörter ohne Suffix betonen die letzte Silbe, die dann auch die letzte Stammsilbe ist. Dies ist vor allem belegbar von Verben im Stativ und Imperativ (naši "trägt", alík „geh!") sowie von einzelnen anderen Wortarten (matí 
„wann?"). Bei Nomina im status constructus ( rabi „groß“) und Präpositionen (qadi „mit") ergibt sich zunächst nur, dass der Akzent nicht auf der Pänultima lag (nicht * rábi). Er lag also entweder auf der Ultima (rabí), oder diese Ausdrücke waren als ganzes unbetont und hatten nur einen Wortgruppenakzent auf dem folgenden Zweitnomen ( $r a b i$ - - ); für letzteres sprechen die Existenz verkürzter status constructus-Formen wie rab (GAG \$64i) sowie die Verhältnisse in anderen semitischen Sprachen. Der Ortsname Kaniš (Nashef 1991: 65-69; = hethitisch Neša) muss entweder endbetont gewesen sein (Kaníš), oder (so Vorschlag Daniel Schwemer) das - $a$ - ist nur eine keilschriftliche Behelfsgraphie für ein eigentlich gesprochenes /kniš/ in diesem wohl fremdsprachlichen Namen.

(2) Kasusendungen sind nicht betont, sondern die unmittelbar vorangehende Silbe, also die letzte Stammsilbe: kúl-u „jeder“; qaqqúr-u „Boden“, Akk. qaqqár-a, Gen. qaqqír-i / qaqqér-e (UGM \$7; CAD Q 117-120); šad(d)u'(')útum, „Abgabe“,Akk. šad(d)u'(')át-am, Gen. šad(d)u'(')ít-im (GKT \$10a); raqqútum (eine Art Stoff), Akk. raqqát-am, Gen. raqqit-im (CAD R 169); nam'ád-am „Mehrzahl (Akk.)", Gen. nam'éd-im (CAD N I 207); simún-u "Zeit" (= babylon. simanu), Gen. simín-i (CAD S 270); arúb-u „Araber“, Gen. Pl. aríb-ī (Eph'al 1982: 6f.; Fales \& Postgate 1992: 235); aššút-u „Ehefrau“, Akk. aššát-a (CAD A II 463); ellút-u „Karawane“, Gen. ellit-i (CAD I/J 83); maškúr-u „Wasserschlauch“, Gen. maškír-i (CAD M I 374); maškún-ū „Zelte (Nom. Pl.)“, Gen. Sg. maškín-i (CAD M I 372); maklúl-u (Art Kleidungsstück), Gen. Pl. maklil-ī-šunu (CAD M I 137); nērúb-u „Durchgang“, Gen. nēríb-i, Pl. nērabāni (CAD N II 176f.; Lanfranchi \& Parpola 1990: 231); kūdún-u „Maultier“, Gen. kūdìn-(i), Pl. kūdín-ī (CAD K 491f.); nāmúr-u „Turm; Spiegel“, Gen. und Pl. nāmélír-i (CAD N I 219f.); mūšéb-ī „Sitze“ (CAD M II 252); ahūzét-e „Ehe (Gen.)“ (CAD A I 217).

(3) Auch Possessivsuffixe werden nicht betont: $p u ́$-šu „sein Mund“. Der Ton liegt auch hier unmittelbar vor dem Suffix, also auf der letzten Stammsilbe, was gegebenenfalls der finale Vokal des status constructus ist: libbá-ka „dein(m) Herz“, libbú-šu „sein Herz“, libbi-ki „dein(f) Herz“, libbí-ni „unser Herz" (CAD L 169f.); dullá-ka „deine Arbeit“, dullú-šu „seine Arbeit“, dullí$n n i$ „unsere Arbeit“ (SNAG \$3.9.6); têrtá-ka „deine Nachricht", têrtú-šu „seine Nachricht", têrti-ni „unsere Nachricht“ (CAD T 358f.); tuppá-ka „deine Tontafel“, tuppú-šu „seine Tontafel“ (GKT §64d); napaští-ni „unser Leben“ (GKT $\$ 64 d$ ); $m e r$ 'it $i$, "meine Tochter“ (CAD M I 301). Vor den pluralischen Suffixen -kunu und -šunu bleibt die Vokalharmonie meist aus, vermutlich weil sie als - $[k n u]$ bzw. - [šnu] gesprochen wurden, was die Silbe geschlossen hat (vgl. Graphien wie tup-pá-áš-nu „ihre Tontafel“ in Michel \& Garelli 1997: Nr. 120a.5 und GKT \$44a sowie weiter Deller 1959: 146-151; GAG \$65f Nachtrag; Hecker 2000: 266; Hirsch 1967: 325; SNAG \$3.9.6).

(4) Sind gleichzeitig Kasusendungen und Possessivsuffixe vorhanden, so liegt der Ton, wie zu erwarten, vor beiden: kúl-u-šu „alles von ihm“ (GKT \$62b); 
ramin-i-(j)a (< ramán-i-ja) „meiner selbst“ (GKT \$10c; Cancik-Kirschbaum 1996: 226); aššit-i-šu „seiner Ehefrau“ (CAD A II 463); mer'it-i-ša „ihrer Tochter" (Michel \& Garelli 1997: Nr. 84.6); kurummit-i-šu „seiner Ration“ (CAD K 577); simén-i-ja „meiner Zeit“ (CAD S 269); qaqqíd-i-ka „deines Kopfes" (CAD Q 104); kima qaqqíd-i-kunu „wie ihr selbst" (CAD Q 106).

(5) Auch das Nisbesuffix dürfte unbetont gewesen sein, wie die folgenden Beispiele zeigen: šubír-i’-im „des Subaräerlandes“ (Nashef 1991: 108f.); akkid-i'- $\bar{e}$ „der Akkader (Gen. Pl.) (Nashef 1991: 5)“ (evtl. nicht aussagekräftig, vgl. unten Abschnitt 7); takšin- $\hat{u}\left(<{ }^{*}\right.$ takšán-i-u) „der aus (der hethitischen Ortschaft) Takšana" (Nashef 1991: 112).

(6) Spezialfälle bis hierher: In Wörtern, in denen der Umlaut einen vermuteten Langvokal zu verändern scheint, ist wohl der Ansatz des Vokals als Länge nicht richtig (vgl. GAG $\$ 15 \mathrm{~d}$ ), so in $a w u ́ t-u$ (geschrieben mit -WA- oder -bu-) „Wort", Gen. awit-i (geschr. mit -WA- oder - bi-) (CAD A II 29-43; traditioneller Ansatz *awätum) und in ramín-i „mich selbst“ (GKT \$64b).

Als zusätzliche Regel muss angenommen werden, dass wortanlautendes $a$ nicht der Vokalharmonie unterliegt. Dies zeigen die Nomina $a b u$ „Vater", $a h u$ „Bruder" und $a h u$ „Arm", deren $a$ - stets erhalten bleibt. Aufschlussreich ist hier der Personenname Šalim-ahum „Wohlbehalten-ist-der-Bruder“, der in assyrischen Texten durchaus in dieser Form erscheint, daneben aber auch in Varianten mit Vokalharmonie, die offenbar möglich wurden, wenn der Name univerbiert und $a h u$ nicht mehr als initial aufgefasst wurde: Genitiv geschrieben als ša-lim-mi $-h i$-im $\sim \check{s} a l_{2}-m i_{3}-h i$-im, d.h. [šal(i)míhim] (Hecker et al. 1998: 398, Michel \& Garelli 1997: 321, Stephens 1944: 15); auf einem Nominativ beruhende Form ohne Kasusendung ša-lim-mu-uh $h_{2} \sim \check{s} a-a l-m u-u h_{2}$, d.h. [šal(i)múh(um)] (Balkan 1974: 37).

In zwei Fällen ist ein Umlaut $a>e / i$ auch vor einer Konsonantengruppe $\mathrm{C}+r$ belegt. Falls es sich hier wirklich um Vokalharmonie handeln sollte, wäre mit Postgate (1974: 274 Anm. 3) zu erwägen, dass die Silbengrenze vor die Konsonantengruppe fallen und sich so auch hier eine offene Silbe ergeben konnte (vergleiche die Regel über muta cum liquida in der lateinischen Metrik): ana $a s ̌ r-i-s ̌ u ~ \sim ~ i s ̌ r-i-s ̌ u ~(=i|s ̌ r i| s ̌ u ?)$,an seinen Platz" (GKT \$10c); adre edre als Gen. von adru „Tenne“ (UGM \$8.2). Allerdings besteht ein Widerspruch zu der oben formulierten Regel, dass die Vokalharmonie wortinitiales $a-$ nicht erfasst.

Der inkonsistent notierte Vokal, der gelegentlich vor $r$ geschrieben wird (GAG \$12b Anm.), unterliegt nicht der Vokalharmonie und dürfte unbetont und vielleicht subphonematisch gewesen sein: zakar-ēe (=[zákərē $]$ ?) „Männer (Gen.Pl.)“ neben Nom.Sg. zakr-um (CAD Z 23); nakar-i nakir-i (= [nákəri]?) „des Feindes" (CAD N I 193); nakar-ūti „der feindlichen“ (CAD N I 193); nukaribb-u „Gärtner“, altassyr. Akk. nukiribb-am (CAD N II 323), Gen. Pl. $n u k r i b b-\bar{\imath}$ (CAD N II 327). Während solche Fälle im älteren Assyrisch seltener als im Babylonischen vorkommen, nehmen im Neuassyrischen geschriebene 
anaptyktische Vokale vor $r$ und anderen Konsonanten sprunghaft zu (Luukko 2004: 102-109).

(7) Nomina mit der äußerst seltenen Stammbildung -CCVCV(C) scheinen nicht die erwartete Endbetonung, sondern einen Pänultimaakzent zu haben: aštipir-um "Gesinde" (einmal altassyrisch, CAD A II 474; = babylonisch aštapir-u); šaluššini „vorletztes Jahr“ (neuassyrisch, CAD Š I 285; = babylonisch šaluššani). Ich würde diese vereinzelten Formen nicht als aussagekräftig einschätzen, gäbe es nicht eine Parallele im Verbalsystem (dazu unten $\mathrm{Ab}$ schnitt 10).

(8) Einige Wörter zeigen wie Vokalharmonie erscheinende Vokalwechsel in Silben, die sowohl nach der hier vertretenen als auch nach der traditionellen Auffassung vortonig sind, z.B.: asītu "Turm“, neuassyr. auch isītu (CAD A II 332f.); dalīlu „Ruhm“, neuassyr. auch dilīlu (CAD D 50f.); kalūmu „Lamm“, altassyr. Akk. kulūmam (CAD K 106); šamuttu šumuttu (eine Pflanze) (Röllig \& Tsukimoto 1999: 436f.; mittelassyrisch); zakûtu „Anweisung“, einmal altassyr. $z u k \hat{u}(t)$-sa (CAD Z 154); zaqīpu „Pfahl“, neuassyr. auch ziqīpu (CAD Z 58). Solche Graphien, die charakteristischerweise nicht konsequent, sondern nur als Variantenschreibungen auftreten, sind wohl nicht mit der Vokalharmonie in Verbindung zu bringen. Es gibt ebensolche graphischen Varianten auch in der Weise, dass eine Erklärung als Vokalharmonie nicht in Betracht kommen kann (vgl. GKT \$8-9; UGM \$8-10; SNAG \$2.4.1; Luukko 2004: 82-88). Ebenfalls von der Vokalharmonie zu trennen sind die Fälle von Angleichung der Auslautvokale an die Pänultima im Neuassyrischen, die Deller \& Parpola (1967) behandelt haben.

(9) In Verbalformen liegt der Ton, soweit beurteilbar, wie bei Nomina meist auf der letzten Stammsilbe, während Suffixe unbetont bleiben. G-Stamm Präteritum: išal „er fragte“, Pl. išúl-u (GKT \$91c); iklú’u „sie hielten zurück“ (GKT \$10a); aṣbút-u „was ich ergriff“ (GKT \$79b), ișbút-u-ma „sie ergriffen“, iṣbút-u-niati-ma (CAD Ș 11); ișbít-i „er ergriff mich“ (Michel \& Garelli 1997: Nr. 180.2); ašam „ich kaufte“, Subj. ašum-u (CAD Š I 351-353). G-Stamm Präsens: išaqqal „er bezahlt“, Pl. išaqqúl-u, ebenso išaqqúl-u-nim (CAD Š II 4); iša” "al „er fragt", Pl. iša”úl-u (UGM \$74.3), ebenso iša"úl-u-ma (CAD Š I 277) und iša"úl-u-šu (CAD Š I 276); ikaššad „er kommt an“, Pl. ikaššúd-u (Cancik-Kirschbaum 1996: 222); iddan „er gibt“, Pl. iddún-u (GKT $\$ 100$ b; UGM \$82.3); illak „er geht“, Pl. illúk-u (UGM \$82.1); išalla „er tut Unrecht“, Pl. išallü'-u (GKT \$95b); inașsar „er bewacht“, Pl. inașúr-u, neuassyrisch auch inassúr (CAD N II 35; SNAG \$2.4.7); tara”am „du(m) liebst“, tara” $i m-i-n i$ „du(f) liebst mich“ (GKT \$91b); tašaqqíl-i-ni „(soviel) du (f) be-

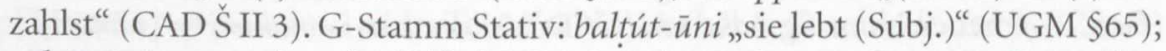
usbút-ūni „sie wohnt (Subj.) (UGM \$78.3). Gt-Stamm Präsens: ištappúk-u „sie deponieren“ (CAD Š I 420); iltappút-u „(der) aufschreibt“ (CAD L 89); ittallúk-u-nim „sie kommen zurück“ (CAD A I 322); iltabbúš-u-niš-šunu „sie werden (die Kleider) tragen“ (CAD L 18). Gtn-Stamm Präsens: ištanappúr- 
$u$-nik-ku-ni „(was) sie dir immer wieder schreiben“ (GKT \$87a). D-Stamm Präsens: uššar „er lässt los“, Pl. uššúr-u (GKT \$93f; UGM \$78.4); ugammúr „sie werden beenden“ (neuassyrisch verkürzt aus * ugammúr-u) (Parpola 1987: Nr. 80). Š-Stamm Präsens: ušettaq „er bewegt“, Pl. ušettúq-u (CAD E 391); ušeššab „er lässt wohnen“, Pl. ušeššúb-u (UGM \$78.5); ušallúm-u „sie bringen in Ordnung“ (UGM \$68.1). N-Stamm Präsens: innammúr- $u$,er wird gesehen (Subj.)" (CAD A II 24).

(10) In bestimmten Verbalformen mit $t$ - oder $n$-Infix, welche die Silbenstruktur-CCVCV (C) haben, lässt sich jedoch ein Akzent auf der vorletzten Stammsilbe nachweisen. Dies betrifft: G-Stamm Perfekt: ( ${ }^{*}$ iqtábi $>$ ) iqtíbi „,er hat gesagt" (UGM \$80.3), ebenso Pl. iqtíbi-u (CAD Q 27); ibtíri ,er hat gehungert“ (CAD B 118); iptíqid „er hat zugewiesen“ (CAD P 120), ebenso iptíqid-su (CAD P 122); ittíziz „er hat gestanden“ (GKT \$100c); tartíši-šum „sie hat für ihn empfangen“ (CAD R 194); iptúhur „er hat sich versammelt" (CancikKirschbaum 1996: 224); tartúgum „sie hat gerufen“ (UGM \$67); addúbub, ,ich habe gesprochen“(CAD D 11); attúmuš,,ich bin aufgebrochen“ (CAD N I 221); ittídin „er hat gegeben“, dagegen Pl. ittadn-u (GKT \$100b); ittísi „er ist fortgegangen“ (GKT \$97e), ebenso tattísi-ma (UGM \$81.5), dagegen Pl. ittaș’u (GAG \$106m) ittas-u (Cancik-Kirschbaum 1996: 229); altéme „ich habe gehört" (Cancik-Kirschbaum 1996: 227); iltéqe'-u ,sie haben genommen“ (UGM $\$ 80.3$ ). Gt-Stamm Präteritum: è taštúpu, du sollst nicht schweigen“ (GKT §95f; weitere ähnliche Belege CAD Š I 491). Gtn-Stamm Präsens: iqtíniap „er vertraut immer wieder an“ (GKT \$94c). N-Stamm Präteritum: ( ${ }^{*}$ inšákin $>$ ) išš́kin „er wurde gelegt“ (CAD Š I 155), ebenso iššikin-ma (CAD Š I 156); libbiši „er soll verfügbar sein“, ebenso Pl. libbíši -u (CAD B 157f.); linnídi „er soll gelegt werden“, ebenso linnídi-ma und innídi'-ūni (CAD N I 99f.); inníši „er wurde getragen“ (CAD N II 111); lattikil „ich will vertrauen“ (CAD T 67); imméger „er einigte sich“ (UGM \$70.3).

(11) Es stellt sich jetzt die Frage, ob diese Pänultimabetonung durch eine morphologische oder durch eine phonologische Regel verursacht ist, ob also durch die Zugehörigkeit zu einem bestimmten Paradigma oder allein durch die Silbenstruktur-CCVCV(C). Aufschlussreich hierfür sind Gt-Formen von Verben primae infirmae, die an Stelle der Doppelkonsonanz einen Langvokal haben.

Das typischerweise im Gt-Stamm gebrauchte Verb awû "sprechen" ist im Altassyrischen gut belegt und zeigt dort meist keine Vokalharmonie (GKT \$99): $e-t a-W A \sim e-t a-W A-u_{2}$ (d.i. wohl [ètawú]) „er sprach“, $n e_{2}$-ta-WA (entweder doch mit Vokalharmonie oder ungenau geschrieben:) $n e_{2}-t u_{3}-u_{2}$,wir wollen sprechen". Dies ein starkes Argument dafür, dass für den Pänultimaakzent solcher Formen wie iqtíbi nicht die morphologische Kategorie, sondern die Silbenstruktur verantwortlich ist.

In die andere Richtung weist nun zwar das Perfekt des G-Stamms von Verben primae infirmae, das bei ebendieser Silbenstruktur gewöhnlich (schein- 
bar?) Vokalharmonie zeigt: èteli „er ist hinaufgegangen“, ebenso Pl. èteli'-u (CAD E 116; Cancik-Kirschbaum 1996: 220); èteriš „er hat gefordert“ (CAD E 282); êtesid „er hat geerntet" (CAD E 340); ètezib-šu „er hat ihn verlassen“" (CAD E 416); tètetiq „sie hat überquert" (CAD E 384). Doch betreffen alle mir bekannten derartigen Belege Verben der $e$-Klasse, die im Babylonischen ebenso lauten müssten. Aussagekräftiger wären Belege von Verben mit $u$-Vokalismus, für die mir kein Beispiel bekannt ist (GAG Paradigma 15 zitiert oder konstruiert eine mir nicht nachweisbare assyrische Form êtušuš „er hat sich betrübt“). Ich halte es daher für möglich, dass in den genannten Fällen gar keine assyrische Vokalharmonie, sondern vielmehr ein $e$-Umlaut nach babylonischem Vorbild vorliegt.

Das Präteritum des N-Stammes von Verben primae infirmae zeigt auffälligerweise keine Vokalharmonie: innamer(-ma) „er wurde gesehen“ (altassyr., GKT \$89d; wir erwarten * innímir); innabit „er floh “ (mittelassyr., CAD A I 46); außer wieder bei einem Verb der $e$-Klasse: linne/ipiš „er soll getan werden“ (GKT \$90d). Es scheint, dass die Vokalharmonie hier zugunsten einer Markierung der Differenz zwischen $a$ - und $e$-Klasse außer Kraft gesetzt ist, also Analogie (Einfluss etwa der Infinitive amāru „sehen“ vs. epāšu „tun“) das Lautgesetz durchbrochen hat. Reguläre Vokalharmonie finden wir hingegen in $a$ "idir-ma ,ich regte mich auf“ (CAD A I 105f.; N-Stamm Präteritum mit starkem Aleph).

Obwohl das Bild kompliziert bleibt, halte ich es für wahrscheinlich, dass der Pänultimaakzent ursprünglich grundsätzlich bei allen Stämmen der Gestalt -CCVCV(C) angebracht war, die nur zufällig fast ausschließlich im Verbalsystem vorkommen. Diese Gesetzmäßigkeit wurde dann durch verschiedenartige Analogiebildungen verwischt.

(12) Wir haben gesehen, dass Suffixe normalerweise unbetont bleiben. Die im Assyrischen häufige Suffixfolge - $\bar{u}$ i $(m)$ (Subjunktiv / Ventiv beim Verb) löst aber in vielen Fällen keine Vokalharmonie aus, was dafür spricht, dass der Akzent unter noch näher zu untersuchenden Bedingungen auf diese schwere Endung verschoben werden konnte: illúk-u illak-ūni (also wohl illakúni) „sie gehen und kommen“ (Lanfranchi \& Parpola 1990: Nos. 35,34f. und 113,13); errúb- $u$,sie treten ein“"vs. Subjunktiv errab-ūni (UGM \$73.2); urrúd-u „sie gehen hinab“ vs. urrad-ūni (UGM \$78.3); ikaššad-ūni „(wenn) er erhält" (GKT $\$ 79 \mathrm{~g}$ ). Diese Fälle wurden schon mehrfach in der Literatur diskutiert (Deller 1959: 71f.; Fabritius 1995; Luukko 2004: 93-97; Postgate 1974: 274; SNAG §2.4.6). Für Postgate waren genau diese Fälle der Anlass, an der gängigen Erklärung der assyrischen Vokalharmonie zu zweifeln, weil das Ausbleiben derselben im Typ illakūni nach der Formulierung der Regel durch GAG unerklärt bleibt.

(13) Mutmaßliche Proklitika wie ša, ana, ina zeigen niemals eine vokalharmonische Angleichung an das Folgewort, was sich im Rahmen der hier vorgeschlagenen These leicht durch ihre Tonlosigkeit erklärt. 
Eine Generalisierung des Befundes ist nicht ganz einfach, weil wir für die zahlreichen Stammbildungstypen, in denen die Voraussetzungen für Vokalharmonie fehlen (z.B. iprus, parāsu, kalbu), auch jetzt keinen direkten Hinweis auf den Akzent gewinnen. Die meisten der aussagekräftigen Fälle lassen aber den Schluss zu, dass man im Assyrischen vorwiegend die letzte Stammsilbe eines Wortes vor Kasus- und sonstigen Suffixen betonte außer in Stämmen der Silbenstruktur-CCVCV $(\mathrm{C})$, bei denen man die vorletzte Stammsilbe betonte. Einen überwiegenden Ultimaakzent für das Akkadische hat schon einmal Zimmern (1897) vorgeschlagen, jedoch ohne aus heutiger Sicht überzeugende Argumente.

Damit liegt der assyrische Akzent näher als bisher vermutet an dem des Hebräischen (nach der tiberiensischen Tradition), z.B. akk. baní „ist gebaut“ = hebr. baná; akk. parís "ist geteilt“ = hebr. parás, akk. iprús (wenn so korrekt generalisiert aus iprús-u) $=$ hebr. yifrós, was auch ein neues Licht auf die Rekonstruktion des Akzents im Ursemitischen werfen könnte. Die aus strukturalistischer Sicht wichtige Frage, ob der Wortakzent im Assyrischen distinktiv war oder nicht, lässt sich noch nicht endgültig beantworten.

Man wird vorerst mit einem Urteil darüber vorsichtig sein müssen, ob die hier für das Assyrische gewonnenen Ergebnisse auf das Akkadische als Ganzes verallgemeinert werden können. Manche haben die Plene-Schreibung, die besonders in bestimmten altbabylonischen Texten sehr häufig ist, mit dem Akzent in Verbindung gebracht. Basierend auf dieser Hypothese, die aber nicht der communis opinio entspricht, rekonstruiert Knudsen (1980: 13-15) für ein altbabylonisches Korpus einen Wortakzent, der etwa in der Mitte zwischen dem Standardmodell und dem hier vorgeschlagenen assyrischen Akzentmodell steht (nach Knudsen z.B. išpur „er hat geschickt" vs. Pl. išpúrū). Es ist durchaus so, dass ein Teil der bisher als auffällig oder irregulär angesehenen babylonischen Pleneschreibungen (neben Knudsen vgl. etwa noch Aro 1953 und 1971: 248-252; Deller 1959: 178f.; GKT \$63k; Hutter 1985: 13f.; Tropper 1998) sich in Silben findet, die nach der hier vertretenen Ansicht im Assyrischen betont wären, doch ergibt sich keineswegs ein konsistentes Bild. Eine weitere mögliche Quelle für die Rekonstruktion des akkadischen Akzents könnten die zahlreichen babylonischen poetischen Texte darstellen. Es wird abzuwarten sein, ob sich all die verschiedenen Bausteine zu einem plausiblen Gesamtmodell des akkadischen Wortakzents werden zusammenfügen lassen.

\section{Bibliographie}

J. Aro 1953: „Abnormal Plene Writings in Akkadian Texts“, StOr 19/11

1971: Rezension zu R. Frankena, Briefe aus der Leider Sammlung \& F.R. Kraus,

Briefe aus dem Archive des Šamaš-Ȟazir, OLZ 66: 246-252 
K. Balkan 1974: „Cancellation of Debts in Cappadocian Tablets from Kültepe“, in K. Bittel et al. (Hrsgg.), Anatolian Studies Presented to Hans Gustav Güterbock on the Occasion of his 65th Birthday, Istanbul 1974: 29-41

G. Buccellati 1997: „Akkadian and Amorite Phonology“, in A.S. Kaye (Hrsg.), Phonologies of Asia and Africa, Winona Lake 1997, Bd. 1: 3-38

$\mathrm{CAD}=$ The Assyrian Dictionary of the Oriental Institute of the University of Chicago, Chicago $1956 \mathrm{ff}$.

E. Cancik-Kirschbaum 1996: Die mittelassyrischen Briefe aus Tall Šèh Hamad, Berlin

K. Deller 1959: Lautlehre des Neuassyrischen, Wien (unpubl. Diss.)

K. Deller \& S. Parpola 1967: „Progressive Vokalassimilation im Neuassyrischen“, Or 36: $337 \mathrm{f}$.

I. Eph'al 1982: The Ancient Arabs. Nomads on the Borders of the Fertile Crescent 9th5th Centuries B.C., Leiden

K. Fabritius 1995: „Vowel Dissimilation as a Marker of Plurality in Neo-Assyrian“, JCS 47: 51-55

F.M. Fales \& J.N. Postgate 1992: Imperial Administrative Records, Part I: Palace and Temple Administration (SAA 7), Helsinki

$\mathrm{GAG}=\mathrm{W}$. von Soden, Grundriss der akkadischen Grammatik, Roma $1995^{3}$

GKT $=$ K. Hecker, Grammatik der Kültepe-Texte, Roma 1968

K. Hecker 2000: „i oder $\bar{\imath}$ im Status constructus?“, AoF 27: 260-268

K. Hecker \& G. Kryszat \& L. Matouš 1998: Kappadokische Keilschrifttafeln aus den Sammlungen der Karlsuniversität Prag, Praha

H. Hirsch 1967: Rezension zu A. Ungnad, Grammatik des Akkadischen, ZA 58: 324-327 1972: „Zum Altassyrischen“, Or 41: 390-431

J. Huehnergard 1997: A Grammar of Akkadian, Atlanta

M. Hutter 1985: Altorientalische Vorstellungen von der Unterwelt. Literar- und religionsgeschichtliche Überlegungen zu 'Nergal und Ereškigal', Freiburg $(\mathrm{CH})$

E.E. Knudsen 1980: „Stress in Akkadian“, JCS 32: 3-16

A. Lancellotti 1962: Grammatica della lingua accadica, Jerusalem

G.B. Lanfranchi \& S. Parpola 1990: The Correspondence of Sargon II, Part II (SAA 5), Helsinki

J. Lewy 1921: Das Verbum in den 'altassyrischen Gesetzen', Diss. Berlin

M. Luukko 2004: Grammatical Variation in Neo-Assyrian, Helsinki

C. Michel \& P. Garelli 1997: Tablettes paléo-assyriennes de Kültepe, Vol. 1, Paris

Kh. Nashef 1991: Répertoire Géographique des Textes Cunéiformes. Band 4: Die Ortsund Gewässernamen der altassyrischen Zeit, Wiesbaden

S. Parpola 1974: „The Alleged Middle/Neo-Assyrian Irregular Verb *naș and the Assyrian Sound Change $\breve{s}>s^{"}$, Assur 1/1: $1-10$

_ 1984: "likalka ittatakku: Two Notes on the Morphology of the Verb alāku in Neo-Assyrian", StOr 55/7

1987: The Correspondence of Sargon II, Part I: Letters from Assyria and the West (SAA 1), Helsinki

J.N. Postgate 1974: Rezension zu W. Mayer, UGM, BiOr 31: 273f.

E. Reiner 1966: A Linguistic Analysis of Akkadian, The Hague

W. Röllig \& A. Tsukimoto 1999: „Mittelassyrische Texte zum Anbau von Gewürzpflanzen", in B. Böck et al. (Hrsg.), Minuscula Mesopotamica. Festschrift für Johannes Renger (AOAT 267), Münster 1999: 427-443

$\mathrm{SNAG}=\mathrm{J}$. Hämeen-Anttila: A Sketch of Neo-Assyrian Grammar, Helsinki 2000 
F.J. Stephens 1944: Old Assyrian Letters and Business Documents, New Haven

J. Tropper 1998: „Pleneschreibung von Pausalformen in den Amarnabriefen EA 9 und EA 11 aus Babylon“, NABU 1998/108

UGM = W. Mayer: Untersuchungen zur Grammatik des Mittelassyrischen, Kevelaer 1971 H. Zimmern 1897: „Ueber Rhythmus im Babylonischen“, ZA 12: 382-392 